\title{
SIMULATION FOR TRAJECTORY TRACKING OF MULTI- FLEXIBLE-LINK SPACE ROBOT WITH DEADZONE
}

\author{
Huang, X.-Q., ${ }^{* * *}$; Tang, X.-T. ${ }^{* *}, \#$ \& Chen, L. ${ }^{*}$ \\ * School of Mechanical Engineering and Automation, Fuzhou University, Fuzhou, Fujian, 350116, \\ P. R. China \\ ** College of Physics and Electronic Information Engineering, Minjiang University, Fuzhou, Fujian, \\ 350108, P. R. China \\ E-Mail: hxq582p@ 163.com, tang_xt@163.com, chnle@fzu.edu.cn ("Corresponding author)
}

\begin{abstract}
Trajectory tracking is a key step for a free-floating space robot (FFSR) system to complete a space operation. However, the flexibility of links and deadzone in joint input torque decrease the system's trajectory tracking accuracy. The angle tracking for the multi-flexible-link FFSR was discussed to suppress the vibrations of the flexible links and prevent the influence of the deadzone. First, the dynamic equation of the FFSR system was derived. Second, the FFSR system was decomposed into the slow and fast subsystems. Then, for the slow subsystem, a robust control method based on a nominal model and a deadzone estimation compensator were proposed. For the fast subsystem, a linear quadratic regulator optimal method was used to actively suppress vibrations. Finally, experiments were carried out to verify that the FFSR is capable of effectively tracking the desired trajectory. The simulation results show the robustness of the control scheme. It effectively suppressed the vibrations as the vibration modes of the links convergent to zero. This study provides a control scheme for improving the trajectory tracking accuracy of FFSR, which is of practical importance. (Received in July 2018, accepted in September 2018. This paper was with the authors 2 weeks for 2 revisions.)
\end{abstract}

Key Words: Multi-Flexible-Link Space Robot, Deadzone, Trajectory Tracking, Flexible Vibration Suppression

\section{INTRODUCTION}

Free-floating space robots (FFSRs) are widely used in space operations and considered to be one of the most promising devices in on-orbit service missions, such as docking, refuelling, repairing, transporting, rescuing, and removing orbital debris [1]. Accurate motion control of FFSR is essential for successful space operations. The FFSR plays an important role in today's aerospace industry [2]. Therefore, the dynamic modelling and analysis and trajectory tracking of FFSR have attracted great attention. The research on trajectory tracking control methods for FFSR can expand the operating space of the manipulator and improve the onorbit service performance.

The precise trajectory tracking of FFSR requires a complex design of the approach. The development trend in the research of FFSR is the application of flexible manipulators. Although flexible manipulators have faster speed and lower energy consumption than rigid ones, the flexible vibrations of manipulators greatly affect control accuracy [1]. For the flexible-link FFSR, a mutual coupling between the rigid motion and flexible vibrations of the links and a coupling among the flexible vibrations of each link exist. Therefore, the flexiblelink FFSR is a strongly coupled nonlinear system, and its trajectory tracking control is more complex than that of a rigid-link space robot. Sabatini et al. [3] used the piezoelectric device to suppress vibration, and the vibration of the flexible link space manipulator was reduced during an on-orbit operation. They [3] also designed an active damping strategy and relevant devices. Kumar et al. [4] adopted a control method on the basis of the reduced-order model to suppress vibration and proposed a trajectory tracking method for a flexible space robot in workspace. In addition, deadzone nonlinearity exists in the actuator system of the FFSR, 
which is caused by the insensitivity of physical devices (such as sensors or drivers) to some small signals. Aside from affecting the output error, it also generates a limit cycle oscillation and even causes control failure. Thus, a deadzone control scheme must be designed to eliminate the adverse effects of deadzone, decrease tracking errors, achieve an ideal trajectory tracking performance, and improve the trajectory tracking accuracy of FFSR [5, 6]. However, corresponding research work considering the influence of link flexibility and deadzone on the trajectory tracking performance of the FFSR is lacking. Therefore, studying the control of the FFSR in the case of link flexibility and deadzone in joint input torque is of great theoretical and practical importance.

\section{STATE OF THE ART}

In the research of FFSR control, trajectory tracking and path planning for rigid-link FFSR have attracted the attention of experts and scholars [7]. Xie et al. [8] proposed a path planning algorithm on the basis of the Gauss-Newton iteration for the motion trajectory of space manipulators. In accordance with the optimal sinusoidal trajectory parameters, the path planning of the space manipulator was achieved. Some scholars have studied a rigid-link FFSR with flexible appendages. Meng et al. [9] drawn the coupling map on the basis of different scenarios of dynamic coupling, such as modal displacements, joint angles, and mass properties, and proposed a trajectory planning method to suppress structural vibration. However, the main concern is the vibration elimination of flexible appendages, not the flexible arm. With research developments, flexible-link FFSR exhibits lightweight, long arm, and heavy load over rigid manipulators and is receiving increasing attention. Hong et al. [10] used the space manipulator system with a flexible end bar as a research object and adopted the nonlinear feedback control method to obtain the decoupling equation between the joint angles and flexible modes, thereby realizing the trajectory tracking of the system. However, the value of gain matrix for the control method is large, and the micro-vibration caused by the flexible arm was unsuppressed. For a flexible-link FFSR, a dynamic coupling between rigid motion and flexible vibrations and a coupling between the flexible modes of each link exist. Therefore, apart from paying attention to the trajectory tracking, the vibration suppression of flexible links must also be considered to obtain a FFSR with high-precision control performance. $\mathrm{Yu}$ and Chen [11] designed an adaptive trajectory tracking and vibration suppression scheme for multi-flexible-link space manipulators. However, this control method requires that the relationship between system dynamics equation and inertia parameters should be linear, thereby increasing the calculation amount. Zhang and Liu [12] proposed a new nonlinear partial differential equation observer for a flexible two-link manipulator and designed a boundary control scheme to adjust the end effector along the reference trajectory and suppress vibration. However, the proposed observer and scheme depend on the accurate dynamic model. Yang et al. [13] studied the closed-loop dynamics and an adaptive robust trajectory tracking approach of a two-flexible-link space manipulator with unknown but bounded disturbances. However, the inertia parameters of the system are often unknown or uncertain, and the control law needs to know the inertia parameters of the system dynamics equation in advance, thereby resulting in deviations to the control design. Cheong et al. [14] expressed the model uncertainty of a two-arm flexible robot as a parameter perturbation and designed a direct parameter updating law to suppress flexible vibrations. Masoudi and Mahzoon [15] studied the dynamic behaviour and response of a FFSR with two flexible arms in a specific mission and derived the dynamic equation of the space manipulator by using Kane's method. The angular momentum conservation law was utilized to eliminate the kinematic redundancy of the system with a linear-quadratic regulator used as a feedback control to suppress vibrations. However, the abovementioned control schemes require that the 
dynamic model is precise or the relationship between dynamics equation and system inertia parameters is linear, and the influence of deadzone is not taken into account.

The deadzone effects must also be considered to study the high-precision control of FFSR. Therefore, selecting a suitable deadzone compensation method to suppress or eliminate the influence of deadzone nonlinearity on the control of space robot is of great practical importance. Some scholars have regarded deadzone as a model uncertainty with adaptive or neural network methods applied to estimate or approximate it. He et al. [5] counted the deadzone function as an uncertainty and approximated it through a neural network, thereby allowing the robot with input deadzone to meet the control requirements, but the deadzone parameters cannot be adaptively adjusted. Sato et al. [6] regarded the input torque uncertainty as the deadzone phenomenon of the robot manipulator, and the nonlinearity and external disturbances were attenuated by $H \infty$ control performance. The proposed method can ensure that the unknown parameters of the deadzone model are adaptively estimated, and the compensator can be designed without solving the Hamilton-Jacobi-Isaacs equation. Similarly, this control method requires that the system dynamic equation is expressed as a linear function of inertial parameters, thereby intensifying the difficulty in the application of complex models. Some scholars have also taken the manipulator with asymmetric deadzone as the research object with an adaptive regulator for deadzone parameters designed. Han et al. [16] adopted a recurrent cerebellar model articulation controller scheme on the basis of the wavelet basis function. However, the method requires the adaptive adjustment of additional parameters, and the debugging is particularly complicated. Cheong et al. [17] proposed an adaptive fuzzy method on the basis of the dynamic surface for robots with deadzone and other uncertainties and some adaptive laws to compensate for nonlinear deadzone by designing recursive steps, thereby overcoming the drawbacks of the backstepping method. However, the left/right slope and width of deadzone should be estimated, thereby making the scheme design and debugging complicated and difficult. Some scholars have adopted sliding mode control methods to suppress the deadzone effects. Hsu and Kuo [18] proposed an intelligent complementary sliding mode control scheme, which used an index threshold of tracking performance to adjust deadzone parameters. When the tracking performance index was less than the performance threshold, the adjustment process of the deadzone parameters stopped, thereby preventing the instability of the system because of the drift of deadzone parameters caused by excessive training of traditional neural network and achieving an excellent control performance. However, the simulation example model is only a single-link robot. Han and Lee [19] designed a sliding surface with position and velocity errors constrained. For robots with unknown deadzone, this method does not require additional design compensation, and the deadzone disturbance can be suppressed within the predetermined error range by adjusting the gain value only. The structure is simple, and the control is stable. However, the main objects of the aforementioned research are ground-based robots, and the influence of deadzone on the trajectory tracking of space robots is less involved.

The abovementioned studies on manipulators, which considered deadzone, aimed at the rigid links. However, research on the flexibility of a single link and deadzone in joint input torque for the FFSR, especially when considering the flexibility of multiple links, is insufficient. The trajectory tracking and flexible vibration suppression in the case of a multiflexible-link FFSR with deadzone were discussed in this study. On the basis of the singular perturbation theory, the system was decomposed into fast and slow subsystems. For the slow subsystem, a robust control scheme was designed to compensate for the uncertain parameters so as to track the desired angle trajectories of the base and joints. For the fast subsystem, a linear quadratic regulator (LQR) optimal method was used to suppress the vibrations of flexible links. Numerical simulations illustrate the effectiveness of the proposed scheme. 
The rest of this paper is organized as follows: Section 3 describes the dynamic modelling of the multi-flexible-link FFSR, decomposes the dynamic system into fast and slow subsystems on the basis of the singular perturbation method, and designs the deadzone compensator and robust control law of the slow subsystem and the LQR optimal method of the fast subsystem. Section 4 simulates and analyses a FFSR with two flexible links to verify the effectiveness of the control scheme. Section 5 summarizes the relevant conclusions.

\section{METHODOLOGY}

\subsection{Dynamic modelling of a FFSR with multiple flexible links}

The structure of FFSR with multiple flexible links is shown in Fig. 1. The system consists of free-floating base $B_{0}$ and multiple flexible links $B_{i}(i=1, \ldots, n)$. The coordinate $O_{i} x_{i} y_{i}$ of each fragment $B_{i}(i=0,1, \ldots, n)$ is established, and $O_{0}$ coincides with $O_{C 0}$, which is the centroid of $B_{0} . O_{i}(i=1, \ldots, n)$ is the centre of the joints connecting $B_{i-1}(i=1, \ldots, n)$ and $B_{i}, x_{i}$-axis is collinear with $O_{i} O_{i+1}$ for $i=1, \ldots, n$, and $x_{n}$-axis is tangent to $B_{n}$ at $O_{n}$. The distance between $O_{0}$ and $O_{1}$ is $l_{0}$. The length of $B_{i}(i=1, \ldots, n)$ along the $x_{1}$-axis is $l_{i}$. The mass and moment of inertia about the centroid of the base are $m_{0}$ and $J_{0}$, respectively. A translational inertial reference frame $(O-X Y)$ is established by taking the arbitrary point $O$ of the space as the origin.

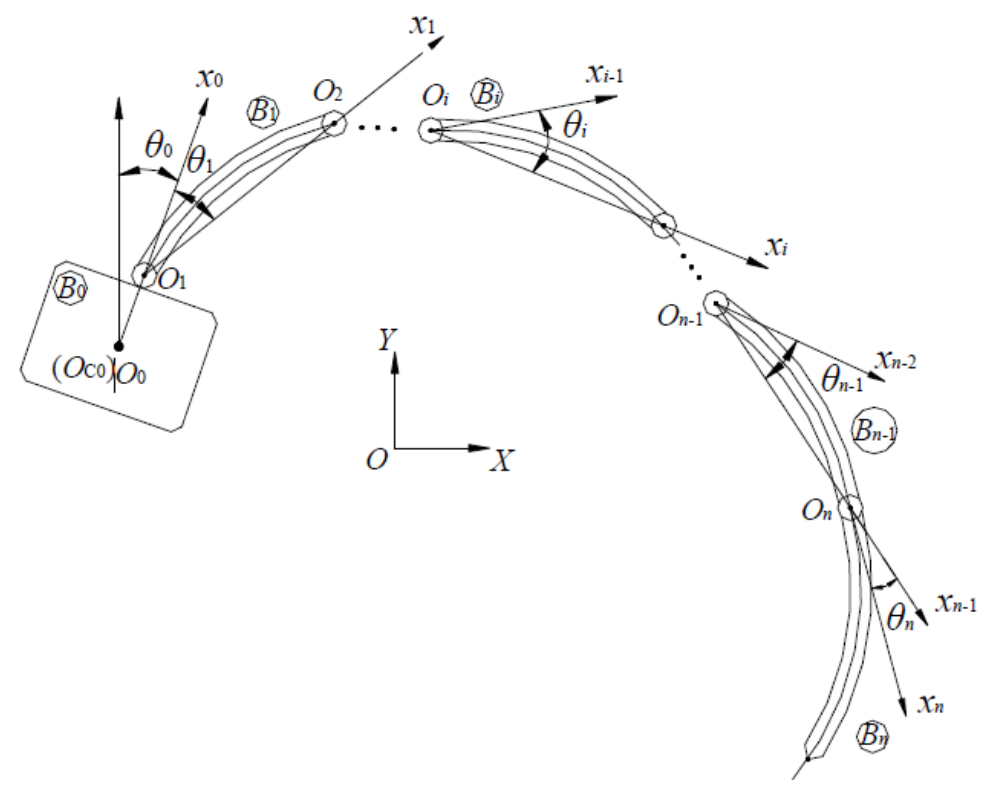

Figure 1: Multi-flexible-link FFSR.

Assumption: 1. Each link is assumed to be long, slender, and homogeneous.

2. The bending deformation is mainly considered, the axial and shearing deformations are negligible, and the transverse vibration is performed in a plane.

The linear density of the flexible link $B_{i}(i=1, \ldots, n)$ is $\rho_{i}$, and the flexural rigidity of the section is $(\mathrm{EI})_{i}$. The flexible manipulators can be regarded as Euler-Bernoulli beams, and their bending deflections can be described as follows:

$$
w_{i}\left(x_{i}, t\right)=\sum_{j=1}^{\gamma_{i}} \varphi_{i j}\left(x_{i}\right) \delta_{i j}(t)
$$

where $w_{i}\left(x_{i}, t\right)$ is the transversal deflection of $B_{i}$ at abscissa $x_{i}\left(0 \leq x_{i} \leq l_{i}\right) ; \varphi_{i j}\left(x_{i}\right)$ is the shape function of the $j^{\text {th }}$ mode of link $i ; \delta_{i j}(t)$ is the time-varying amplitude associated with $\varphi_{i j}\left(x_{i}\right) ; \gamma_{i}$ is the number of mode; and $B_{i}(i=1, \ldots, n-1)$ and $B_{n}$ are regarded as simply supported and 
cantilever beams, respectively. According to the literature [20], the beams' shape functions can be expressed.

According to the law of conservation of momentum and Lagrange's equation of the second kind, the dynamic equation of the FFSR system with multiple flexible links is expressed as follows:

$$
\mathbf{M}(\boldsymbol{\theta}, \boldsymbol{\delta})\left[\begin{array}{c}
\ddot{\boldsymbol{\theta}} \\
\ddot{\boldsymbol{\delta}}
\end{array}\right]+\mathbf{H}(\boldsymbol{\theta}, \dot{\boldsymbol{\theta}}, \boldsymbol{\delta}, \dot{\boldsymbol{\delta}})\left[\begin{array}{c}
\dot{\boldsymbol{\theta}} \\
\dot{\boldsymbol{\delta}}
\end{array}\right]+\left[\begin{array}{c}
0 \\
\mathbf{K} \boldsymbol{\delta}
\end{array}\right]=\left[\begin{array}{c}
\boldsymbol{\tau}_{3 \times 1} \\
\mathbf{0}_{4 \times 1}
\end{array}\right]
$$

where $\boldsymbol{\theta}=\left[\theta_{0} \ldots \theta_{i} \ldots \theta_{n}\right]^{\mathrm{T}} \in \mathbf{R}^{n+1}$ is the generalized coordinate vector of the base attitude and relative joint angles, $\boldsymbol{\delta}=\left[\delta_{11} \cdots \delta_{1 \gamma_{1}} \cdots \delta_{n \gamma_{i}}\right]^{\mathrm{T}} \in \mathbf{R}^{\sum_{i=1}^{n} \gamma_{i}}, \mathbf{M}(\boldsymbol{\theta}, \boldsymbol{\delta}) \in \mathbf{R}^{\left(n+1+\sum_{i=1}^{n} \gamma_{i}\right) \times\left(n+1+\sum_{i=1}^{n} \gamma_{i}\right)}$ is the inertia matrix, and $\mathbf{H}(\boldsymbol{\theta}, \dot{\boldsymbol{\theta}}, \boldsymbol{\delta}, \dot{\boldsymbol{\delta}})\left(\dot{\boldsymbol{\theta}}^{\mathrm{T}} \dot{\boldsymbol{\delta}}^{\mathrm{T}}\right)^{\mathrm{T}} \in \mathbf{R}^{n+1+\sum_{i=1}^{n} \gamma_{i}}$ is the column vector of the Coriolis and centrifugal forces. $\mathbf{K} \in \operatorname{diag}\left(k_{11}, \ldots, k_{n \gamma_{i}}\right) \in \mathbf{R}^{\left(\sum_{i=1}^{n} \gamma_{i}\right) \times\left(\sum_{i=1}^{n} \gamma_{i}\right)}\left(k_{i j}=(\mathrm{EI})_{i} \int_{0}^{l_{i}} \varphi_{i j}^{\prime \prime \mathrm{T}} \varphi_{i j}^{\prime \prime} \mathrm{d} x_{i}\right)$ is the stiffness matrix. $\boldsymbol{\tau}=\left[\tau_{0} \ldots \tau_{i} \ldots \tau_{n}\right]^{\mathrm{T}} \in \mathbf{R}^{n+1}$ is the vector of input torques of the base and joints.

\subsection{Fast and slow subsystems decomposition based on singular perturbation theory}

A FFSR with multiple flexible links deforms and vibrates during movements. In order to achieve the desired trajectory tracking and vibration suppression, the singular perturbation method is considered in this study, and the dynamic system is decomposed into a slow subsystem representing rigid motion and a fast subsystem expressing the vibrations of flexible links. The control law $\bar{\tau}$ of the slow subsystem is designed to ensure the rigid desired trajectory tracking, and the control law $\boldsymbol{\tau}_{f}$ of the fast subsystem is proposed to suppress flexible vibrations.

Then, the combination control law is expressed as follows:

$$
\boldsymbol{\tau}=\overline{\boldsymbol{\tau}}+\boldsymbol{\tau}_{f}
$$

Eq. (2) can be rewritten as block matrixes:

$$
\left[\begin{array}{ll}
\mathbf{M}_{r r} & \mathbf{M}_{r f} \\
\mathbf{M}_{f r} & \mathbf{M}_{f f}
\end{array}\right]\left[\begin{array}{c}
\ddot{\boldsymbol{\theta}} \\
\ddot{\boldsymbol{\delta}}
\end{array}\right]+\left[\begin{array}{ll}
\mathbf{H}_{r r} & \mathbf{H}_{r f} \\
\mathbf{H}_{f r} & \mathbf{H}_{f f}
\end{array}\right]\left[\begin{array}{c}
\dot{\boldsymbol{\theta}} \\
\dot{\boldsymbol{\delta}}
\end{array}\right]+\left[\begin{array}{c}
\mathbf{0} \\
\mathbf{K} \boldsymbol{\delta}
\end{array}\right]=\left[\begin{array}{c}
\boldsymbol{\tau} \\
\mathbf{0}
\end{array}\right]
$$

where $\mathbf{M}_{r r} \in \mathbf{R}^{(n+1) \times(n+1)}, \mathbf{M}_{f f} \in \mathbf{R}^{\sum_{i=1}^{n} \gamma_{i} \times \sum_{i=1}^{n} \gamma_{i}}$ and $\mathbf{M}_{r f}=\mathbf{M}_{f r}^{\mathrm{T}} \in R^{(n+1) \times \sum_{i=1}^{n} \gamma_{i}}$ are the submatrices of $\mathbf{M}(\boldsymbol{\theta}, \boldsymbol{\delta}) . \quad \mathbf{H}_{r r} \in \mathbf{R}^{(n+1) \times(n+1)}, \quad \mathbf{H}_{r f} \in \mathbf{R}^{(n+1) \times \sum_{i=1}^{n} \gamma_{i}}, \quad \mathbf{H}_{f r} \in \mathbf{R}^{\sum_{i=1}^{n} \gamma_{i} \times(n+1)}$ and $\mathbf{H}_{f f} \in \mathbf{R}^{\sum_{i=1}^{n} \gamma_{i} \times \sum_{i=1}^{n} \gamma_{i}}$ are the submatrices of $\mathbf{H}(\boldsymbol{\theta}, \boldsymbol{\delta}, \dot{\boldsymbol{\theta}}, \dot{\boldsymbol{\delta}})$.

Considering that $\mathbf{M}$ is a symmetric and positive definite matrix, its inverse exists and it can be written as follows:

$$
\mathbf{N}=\mathbf{M}^{-1}=\left[\begin{array}{ll}
\mathbf{M}_{r r} & \mathbf{M}_{r f} \\
\mathbf{M}_{f r} & \mathbf{M}_{f f}
\end{array}\right]^{-1}=\left[\begin{array}{ll}
\mathbf{N}_{r r} & \mathbf{N}_{r f} \\
\mathbf{N}_{f r} & \mathbf{N}_{f f}
\end{array}\right]
$$

Define the singular perturbation scale factor $\varepsilon^{2}=1 / \min \left\{k_{11}, k_{12}, k_{21}, k_{22}\right\}$, new state variable $\mathbf{z}=\boldsymbol{\delta} / \varepsilon^{2}$, and new matrix $\tilde{\mathbf{K}}=\varepsilon^{2} \mathbf{K}$. Thus, the singular perturbation model of the FFSR system with multiple flexible links is obtained from Eq. (5):

$$
\ddot{\boldsymbol{\theta}}=-\left(\mathbf{N}_{r r} \mathbf{H}_{r r}+\mathbf{N}_{r f} \mathbf{H}_{f r}\right) \dot{\boldsymbol{\theta}}-\left(\mathbf{N}_{r r} \mathbf{H}_{r f}+\mathbf{N}_{r f} \mathbf{H}_{f f}\right) \varepsilon^{2} \dot{\mathbf{z}}-\mathbf{N}_{r f} \tilde{\mathbf{K}} \mathbf{z}+\mathbf{N}_{r r} \boldsymbol{\tau}
$$




$$
\varepsilon^{2} \ddot{\mathbf{z}}=-\left(\mathbf{N}_{f r} \mathbf{H}_{r r}+\mathbf{N}_{f f} \mathbf{H}_{f r}\right) \dot{\boldsymbol{\theta}}-\left(\mathbf{N}_{f r} \mathbf{H}_{r f}+\mathbf{N}_{f f} \mathbf{H}_{f f}\right) \varepsilon^{2} \dot{\mathbf{z}}-\mathbf{N}_{f f} \tilde{\mathbf{K}} \mathbf{z}+\mathbf{N}_{f r} \boldsymbol{\tau}
$$

For the sake of obtaining the slow subsystem, the singular perturbation model is evaluated with $\varepsilon=0$. Then, the slow subsystem is obtained:

$$
\overline{\mathbf{M}}_{r r} \ddot{\boldsymbol{\theta}}+\overline{\mathbf{H}}_{r r} \dot{\boldsymbol{\theta}}=\overline{\boldsymbol{\tau}}
$$

where the matrix or variable with a bar "一” indicates the corresponding slow variable.

The fast subsystem is obtained by introducing new variables $\mathbf{p}_{1}=\mathbf{z}-\overline{\mathbf{z}}, \mathbf{p}_{2}=\varepsilon \dot{\mathbf{z}}$ and a fast time scale $\varpi=t / \varepsilon$ :

$$
\begin{gathered}
\frac{\mathrm{d} \mathbf{p}_{1}}{\mathrm{~d} \varpi}=\mathbf{p}_{2} \\
\frac{\mathrm{d} \mathbf{p}_{2}}{\mathrm{~d} \varpi}=-\overline{\mathbf{N}}_{f f} \tilde{\mathbf{K}} \mathbf{p}_{1}+\overline{\mathbf{N}}_{f r} \boldsymbol{\tau}_{f}
\end{gathered}
$$

This subsystem describes the vibrations of flexible links.

\subsection{Deadzone compensator and control law design for the slow subsystem}

The output torque of a physical device is zero before the input torque reaches a specific value. This input-output relationship is called deadzone. Based on the control theory, the deadzone indicates a loss of information.

The adjustment of the base attitude and position of FFSR is usually conducted by a momentum wheel or jet thruster. While the joint is driven by a motor, a deadzone exists in the joint input torque. Assuming that $\overline{\boldsymbol{\tau}}_{\mathrm{d}}(t)=\left[\overline{\boldsymbol{\tau}}_{\mathrm{d} 1} \cdots \bar{\tau}_{\mathrm{d} n}\right]^{\mathrm{T}}$ and $\overline{\boldsymbol{\tau}}_{r}=\left[\bar{\tau}_{1} \cdots \bar{\tau}_{n}\right]^{\mathrm{T}}=W\left(\overline{\boldsymbol{\tau}}_{\mathrm{d}}\right)$ are the input and output torques of the deadzone, respectively, then the asymmetric deadzone model is as follows [16]:

$$
\begin{gathered}
\overline{\boldsymbol{\tau}}_{r}=\mathbf{W}\left(\overline{\boldsymbol{\tau}}_{\mathrm{d}}\right)=\overline{\boldsymbol{\tau}}_{\mathrm{d}}-\operatorname{sat}\left(\overline{\boldsymbol{\tau}}_{\mathrm{d}}\right) \\
\operatorname{sat}_{i}\left(\bar{\tau}_{\mathrm{d} i}\right)=\left\{\begin{array}{cc}
d_{i+} \quad \bar{\tau}_{\mathrm{d} i} \geq d_{i+} \\
\bar{\tau}_{d i} & -d_{i-}<\bar{\tau}_{\mathrm{d} i}<d_{i+} \\
-d_{i-} & \bar{\tau}_{\mathrm{d} i} \leq-d_{i-}
\end{array} \quad(i=1, \cdots, n)\right.
\end{gathered}
$$

where $d_{i-}>0$ and $d_{i+}>0$ are the left and right breakpoints of the deadzone, respectively, which are unknown but bounded.

Then, the widths of the deadzone are $\mathbf{D}=\left[\mathbf{d}_{+} \mathbf{d}-\right]^{\mathrm{T}} \in \mathbf{R}^{2 n \times n}, \mathbf{d}_{+}=\operatorname{diag}\left(d_{1+}, \ldots, d_{1+}, \ldots, d_{\mathrm{n}+}\right)$, and $\mathbf{d}-=\operatorname{diag}\left(d_{1}, \ldots, d_{1-}, \ldots, d_{\mathrm{n}-}\right)$. A normal number $D_{M}$ exists, which makes $\|\mathbf{D}\| \leq \mathrm{D}_{M}$.

A deadzone compensator is designed to compensate the adverse effect of the deadzone, whose principle is shown in Fig. 2. After ideal torque $\overline{\mathbf{w}}_{r}$ passes through the deadzone compensator, $\overline{\boldsymbol{\tau}}_{\mathrm{d}}$ is obtained. $\overline{\boldsymbol{\tau}}_{r}$ is the final output torque after $\overline{\boldsymbol{\tau}}_{\mathrm{d}}$ passes through the deadzone. At this time, $\overline{\boldsymbol{\tau}}_{r}$ has eliminated the influence of the deadzone, thereby making $\overline{\boldsymbol{\tau}}_{r}$ consistent with $\overline{\mathbf{w}}_{r}$. The deadzone width $\mathbf{D}$ is unknown with estimation $\hat{\mathbf{D}}=\left[\begin{array}{ll}\hat{\mathbf{d}}_{+} & \hat{\mathbf{d}}_{-}\end{array}\right]^{\mathrm{T}}$ used to offset the deadzone.

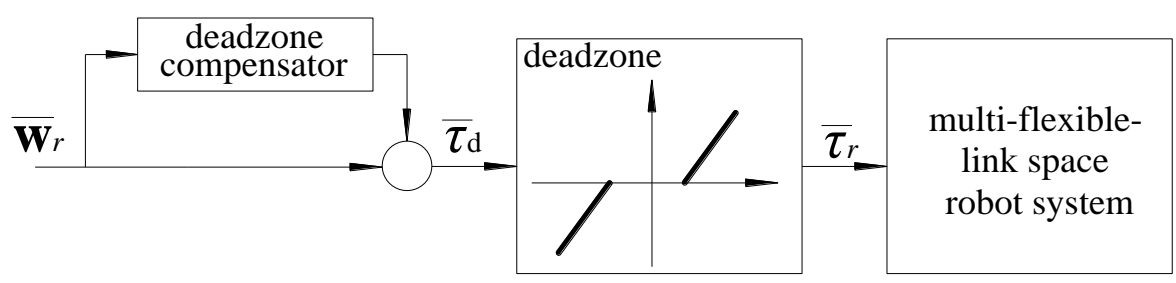

Figure 2: Adaptive deadzone compensation principle. 
Before passing through the deadzone, $\overline{\mathbf{w}}_{r}$ is compensated by the deadzone compensator [21]:

$$
\overline{\boldsymbol{\tau}}_{\mathrm{d}}=\overline{\mathbf{w}}_{r}+\hat{\mathbf{D}}^{\mathrm{T}} \overline{\boldsymbol{\mu}}
$$

where $\overline{\mathbf{w}}_{r}$ is the torque vector before the deadzone compensation, $\overline{\boldsymbol{\mu}}=\left[\begin{array}{llll}\mu_{1} & \mu_{2} & \mu_{1}-1 & \mu_{2}-1\end{array}\right]^{\mathrm{T}}, \mu_{i}=1\left(\bar{w}_{r i} \geq 0\right)$, and $\mu_{i}=0\left(\bar{w}_{r i}<0\right)$.

After the deadzone compensation, $\overline{\boldsymbol{\tau}}_{r}$ can be derived as follows [21]:

$$
\overline{\boldsymbol{\tau}}_{r}=\overline{\mathbf{w}}_{r}-\tilde{\mathbf{D}}^{\mathrm{T}} \overline{\boldsymbol{\mu}}+\tilde{\mathbf{D}}^{\mathrm{T}} \boldsymbol{\chi}
$$

where $\tilde{\mathbf{D}}=\mathbf{D}-\hat{\mathbf{D}}$ is the matrix of the deadzone width estimation error and modelling mismatch $\chi$ satisfies the bound $\|\chi\| \leq \sqrt{n}[21]$.

Assuming that $\boldsymbol{\theta}$ and its velocity $\dot{\boldsymbol{\theta}}$ can be measured, the desired trajectory $\boldsymbol{\theta}_{\mathrm{d}}=\left[\theta_{0 \mathrm{~d}} \ldots \theta_{i \mathrm{~d}} \ldots \theta_{n \mathrm{~d}}\right]^{\mathrm{T}} \in \mathbf{R}^{n+1}$ and its derivative $\dot{\boldsymbol{\theta}}_{\mathrm{d}}$ are determined. The system filter trajectory error is defined as follows:

$$
\mathbf{s}=\Lambda \mathbf{e}+\dot{\mathbf{e}}
$$

where $\boldsymbol{\Lambda} \in \mathbf{R}^{(n+1) \times(n+1)}$ is a symmetric and positive definite constant matrix. $\mathbf{e}=\boldsymbol{\theta}_{\mathrm{d}}-\boldsymbol{\theta}$ is the system tracking error.

The control objective is to design the controller of the slow subsystem $\overline{\mathbf{w}}$, thereby enabling $\boldsymbol{\theta}$ to achieve the desired trajectory with high accuracy.

The robust control law is designed as follows:

$$
\overline{\mathbf{W}}=\hat{\overline{\mathbf{M}}}_{r r} \dot{\mathbf{q}}_{\mathrm{r}}+\hat{\overline{\mathbf{H}}}_{r r} \mathbf{q}_{\mathrm{r}}+\mathbf{k}_{s} \mathbf{s}+\mathbf{w}_{\rho}
$$

where $\overline{\mathbf{w}}=\left[\begin{array}{ll}\overline{\boldsymbol{\tau}}_{0} & \overline{\mathbf{w}}_{r}^{\mathrm{T}}\end{array}\right]^{\mathrm{T}} ; \hat{\overline{\mathbf{M}}}_{r r}$ and $\hat{\overline{\mathbf{H}}}_{r r}$ are the estimated values of $\overline{\mathbf{M}}_{r r}$ and $\overline{\mathbf{H}}_{r r}$, respectively; $\mathbf{w}_{\rho}=\rho \mathbf{S} /\|\mathbf{S}\|$ is a robust control term and $\rho$ is a bounded and positive constant. $\mathbf{q}_{r}=\boldsymbol{\Lambda} \mathbf{e}+\dot{\boldsymbol{\theta}}_{\mathrm{d}}$ is the auxiliary reference output vector.

The error equation can be obtained by combining Eqs. (7) and (11) to (13):

$$
\overline{\mathbf{M}}_{r r} \dot{\mathbf{s}}=\overline{\mathbf{M}}_{r r} \dot{\mathbf{q}}_{r}-\overline{\boldsymbol{\tau}}+\overline{\mathbf{H}}_{r r} \dot{\boldsymbol{\theta}}=\Delta \overline{\mathbf{M}} \dot{\mathbf{q}}_{r}+\Delta \overline{\mathbf{H}} \mathbf{q}_{\mathrm{r}}-\mathbf{k}_{s} \mathbf{s}-\mathbf{w}_{\rho}-\left[\begin{array}{c}
0 \\
\tilde{\mathbf{D}}^{\mathrm{T}} \boldsymbol{\chi}-\tilde{\mathbf{D}}^{\mathrm{T}} \overline{\boldsymbol{\mu}}
\end{array}\right]-\overline{\mathbf{H}}_{r r} \mathbf{s}
$$

where $\overline{\boldsymbol{\tau}}=\left[\begin{array}{cc}\overline{\boldsymbol{\tau}}_{0} & \overline{\boldsymbol{\tau}}_{r}^{\mathrm{T}}\end{array}\right]^{\mathrm{T}}, \Delta \overline{\mathbf{M}}=\overline{\mathbf{M}}_{r r}-\hat{\mathbf{M}}_{r r}$, and $\Delta \overline{\mathbf{H}}=\hat{\mathbf{H}}_{r r}-\overline{\mathbf{H}}_{r r} . \Psi=\Delta \overline{\mathbf{M}} \dot{\mathbf{q}}_{r}+\Delta \overline{\mathbf{H}} \mathbf{q}_{\mathrm{r}}$ is the model error of the system. Assume that $\Psi$ is bounded (i.e., $\|\Psi\| \leq \rho$ ).

Theorem: For the slow subsystem of the FFSR with multiple flexible links, Eq. (7), utilizing the control law, Eq. (13), and the following deadzone width adaptive law, filtering error $\mathbf{s}$ and deadzone width estimate $\hat{\mathbf{D}}$ were eventually uniformly bounded with tracking error e converging to zero in an arbitrarily small neighbourhood.

$$
\dot{\hat{\mathbf{D}}}=-\dot{\tilde{\mathbf{D}}}=\boldsymbol{\Xi} \overline{\boldsymbol{\mu}} \mathbf{s}_{\mathrm{r}}^{\mathrm{T}}-\xi \boldsymbol{\Xi} \hat{\mathbf{D}}\left\|\mathbf{S}_{\mathrm{r}}\right\|
$$

where $\boldsymbol{\Xi} \in \mathbf{R}^{2 n \times 2 n}, \xi>0$, and $\mathbf{s}_{\mathrm{r}}=\left[s_{1} \ldots s_{n}\right]^{\mathrm{T}}$ is the last $n$ terms of $\mathbf{s}$.

Proof: Creating the following Lyapunov function $V$ :

$$
V=\frac{1}{2} \mathbf{s}^{\mathrm{T}} \overline{\mathbf{M}}_{r r} \mathbf{S}+\frac{1}{2} \operatorname{tr}\left(\tilde{\mathbf{D}}^{\mathrm{T}} \mathbf{\Xi}^{-1} \tilde{\mathbf{D}}\right)
$$

Taking the first derivative of $V$ with respect to time $t$,

$$
\dot{V}=\mathbf{s}^{\mathrm{T}} \overline{\mathbf{M}}_{r r} \dot{\mathbf{S}}+\frac{1}{2} \mathbf{S}^{\mathrm{T}} \dot{\overline{\mathbf{M}}}_{r r} \mathbf{S}-\operatorname{tr}\left(\tilde{\mathbf{D}}^{\mathrm{T}} \boldsymbol{\Xi}^{-1} \dot{\hat{\mathbf{D}}}\right)
$$


Considering that $\dot{\overline{\boldsymbol{M}}}_{r r}-2 \overline{\boldsymbol{H}}_{r r}$ is a skew-symmetric matrix [11]; given that $\|\chi\| \leq \sqrt{n},\|\Psi\| \leq \rho$, $\|\mathbf{D}\| \leq \mathrm{D}_{M}$, and $\left\|\mathbf{s}_{\mathrm{r}}\right\| \leq\|\mathbf{s}\|$; and substituting Eqs. (14) and (15), the following inequality is obtained:

$$
\dot{V} \leq-\|\mathbf{S}\|\left[k_{\min }\|\mathbf{S}\|-c_{0}\|\tilde{\mathbf{D}}\|+\xi\|\tilde{\mathbf{D}}\|^{2}\right]
$$

where $k_{\min }=\sigma_{\min }\left(\mathbf{k}_{s}\right)$ is the smallest singular value of matrix $\mathbf{k}_{s}$ and $c_{0} \equiv \sqrt{n}+\xi \mathbf{D}_{\mathrm{M}}$.

As shown in Eq. (18), the condition that $\dot{V}$ is negative is met as long as either $\|\mathbf{s}\|>c_{0}^{2}$ $/ 4 k_{\min } \xi$ or $\|\tilde{\mathbf{D}}\|>c_{0} / \xi$. Therefore, $\dot{V} \leq 0$. According to the expansion of the Lyapunov theory, $\|\mathbf{s}\|$ and $\|\tilde{\mathbf{D}}\|$ are uniformly ultimately bounded (UUB) in the compact set. Therefore, $\mathbf{s}(t)$ and $\hat{\mathbf{D}}(t)$ are UUB (i.e., e converges to zero in an arbitrarily small neighbourhood).

\subsection{LQR optimal method of the fast subsystem}

A fast subsystem is linear and completely controllable regardless of its uncertainties. On the basis of the LQR optimal method, the control scheme for the fast subsystem is designed to actively suppress the vibrations of flexible links.

The fast subsystem, Eq. (8), is expressed as a state equation by setting $\boldsymbol{\sigma}=\left[\begin{array}{ll}p_{1}^{T} & p_{2}^{T}\end{array}\right]^{T}$ :

$$
\dot{\boldsymbol{\sigma}}=\mathbf{A} \boldsymbol{\sigma}+\mathbf{B} \boldsymbol{\tau}_{f}
$$

where $\mathbf{A}=\left[\begin{array}{cc}\mathbf{0}_{\left(\sum_{i=1}^{n} \gamma_{i}\right) \times\left(\sum_{i=1}^{n} \gamma_{i}\right)} & \mathbf{I}_{\left(\sum_{i=1}^{n} \gamma_{i}\right) \times\left(\sum_{i=1}^{n} \gamma_{i}\right)} \\ -\overline{\mathbf{N}}_{f f} \tilde{\mathbf{K}} & \mathbf{0}_{\left(\sum_{i=1}^{n} \gamma_{i}\right) \times\left(\sum_{i=1}^{n} \gamma_{i}\right)}\end{array}\right]$ and $\mathbf{B}=\left[\begin{array}{c}\mathbf{0}_{\left(\sum_{i=1}^{n} \gamma_{i}\right) \times(n+1)} \\ \overline{\mathbf{N}}_{f r}\end{array}\right]$.

As shown in Eq. (19), the fast subsystem is a linear system, and the system state variable $\boldsymbol{\sigma}$ can be adjusted to zero by the optimal control method, thereby suppressing multi-flexiblelink vibrations. For a linear system, if the performance index function is defined as an integral quadratic function of the state and control variables, then control quantity $\boldsymbol{\tau}_{f}$ can be obtained when the function is minimum. Then, the system can obtain the optimal performance.

$$
J=\frac{1}{2} \int_{0}^{\infty}\left(\boldsymbol{\sigma}^{\mathrm{T}} \mathbf{Q} \boldsymbol{\sigma}+\boldsymbol{\tau}_{f}^{\mathrm{T}} \mathbf{R} \boldsymbol{\tau}_{f}\right) \mathrm{d} t
$$

where $\mathbf{Q} \in \mathbf{R}^{\left(\sum_{i=1}^{n} 2 \gamma_{i}\right) \times\left(\sum_{i=1}^{n} 2 \gamma_{i}\right)}$ and $\mathbf{R} \in \mathbf{R}^{(n+1) \times(n+1)}$ are symmetric positive semi-definite and symmetric positive definite weighted matrixes, respectively.

The control scheme should be designed by the LQR theory to minimize $J$ :

$$
\boldsymbol{\tau}_{f}=-\mathbf{R}^{-1} \mathbf{B}^{\mathrm{T}} \mathbf{P} \boldsymbol{\sigma}
$$

where $\mathbf{P}$ satisfies the algebraic Riccati equation $\mathbf{P A}+\mathbf{A}^{\mathrm{T}} \mathbf{P}-\mathbf{P B} \mathbf{R}^{-1} \mathbf{B}^{\mathrm{T}} \mathbf{P}+\mathbf{Q}=\mathbf{0}$.

\section{SIMULATION RESULT ANALYSIS}

In this study, a two-flexible-link FFSR system is taken as an example, and numerical simulation experiments are conducted with deadzone compensator, Eq. (11), trajectory tracking scheme, Eq. (13), a deadzone width adaptive law, Eq. (15), and vibration suppression scheme, Eq. (21). The block diagram of the control system is shown in Fig. 3.

The physical parameters of the system are as follows: $l_{0}=l_{1}=1.5 \mathrm{~m}, l_{2}=1.0 \mathrm{~m}, m_{0}=40 \mathrm{~kg}$, $J_{0}=34.17 \mathrm{~kg} \cdot \mathrm{m}^{2}, \rho_{1}=3.5 \mathrm{~kg} / \mathrm{m}, \rho_{2}=1.1 \mathrm{~kg} / \mathrm{m}$, and $(\mathrm{EI})_{1}=(\mathrm{EI})_{2}=50 \mathrm{~N} \cdot \mathrm{m}^{2}$. 


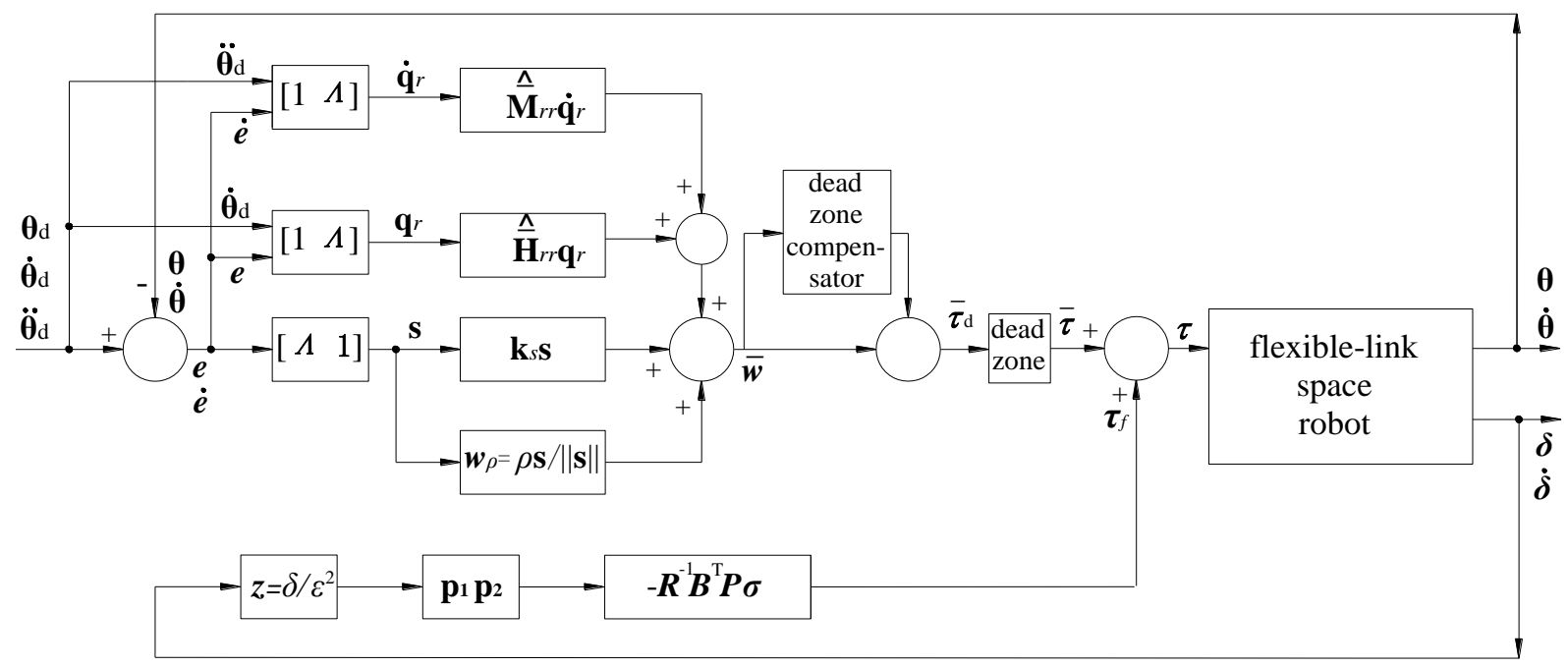

Figure 3: Control block diagram of flexible-link FFSR system.

The calculation is simplified while ensuring the calculation accuracy as much as possible, considering that the large amplitude vibrations are mainly composed of low-order modes and $\gamma_{i}=2$.

The desired trajectories of the system are $\theta_{0 \mathrm{~d}}=0 \mathrm{rad}, \theta_{1 \mathrm{~d}}=\pi / 3 \mathrm{rad}$, and $\theta_{2 \mathrm{~d}}=\pi / 6 \mathrm{rad}$.

The initial state $\boldsymbol{\theta}$ is as follows: $\theta_{0}=-0.4 \mathrm{rad}, \theta_{1}=0.5 \mathrm{rad}$, and $\theta_{2}=\pi / 4 \mathrm{rad}$.

The trajectory tracking time in the simulation is $t=40 \mathrm{~s}$.

The simulation results are shown in the figures. Figs. 4 to 6 show a comparison between the desired angular displacements and the actual ones of the base and two joints, and Figs. 7 to 10 demonstrate the first- and second-order flexible modes of vibrations of the two links when deadzone compensator, Eq. (11), and control laws, Eqs. (13) and (21), are adopted.

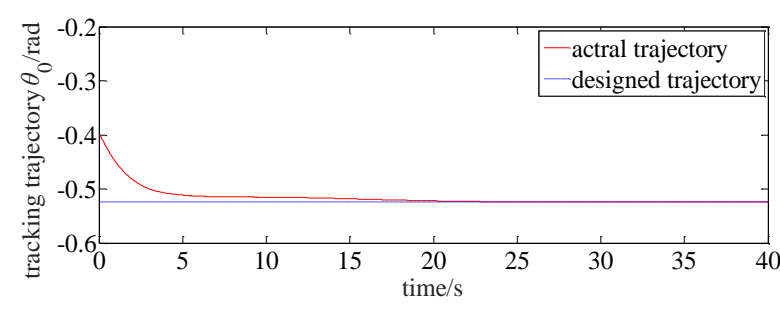

Figure 4: Trajectory tracking of base attitude.

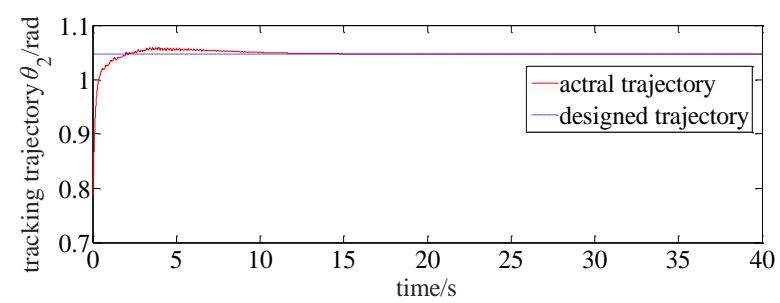

Figure 6: Trajectory tracking of joint 2 angle.

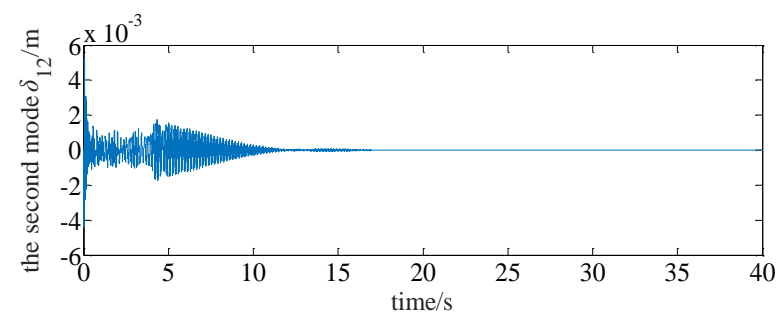

Figure 8: Second-order mode of flexible link $B_{1}$.

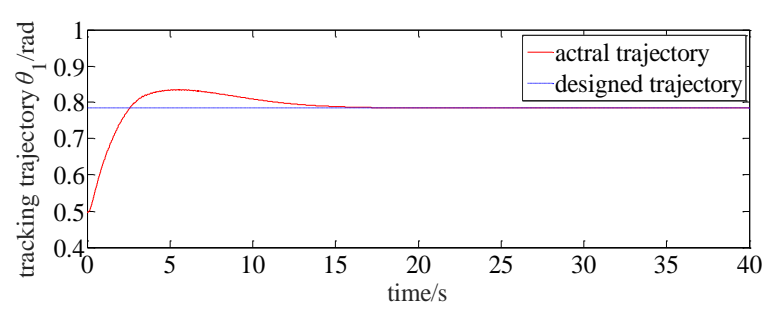

Figure 5: Trajectory tracking of joint 1 angle.

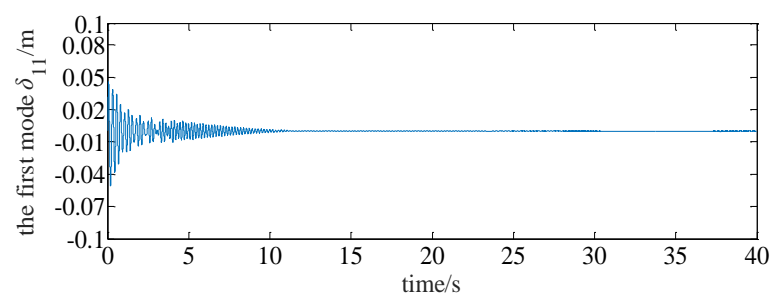

Figure 7: First-order mode of flexible link $B_{1}$.

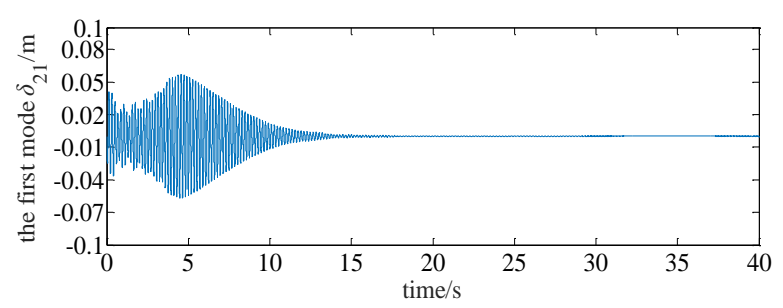

Figure 9: First-order mode of flexible link $B_{2}$. 


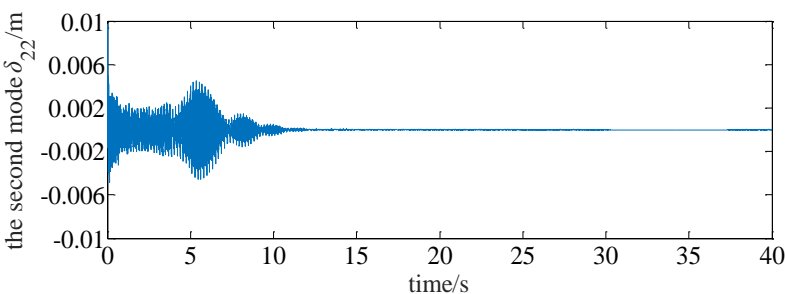

Figure 10: Second-order mode of flexible link $B_{2}$.

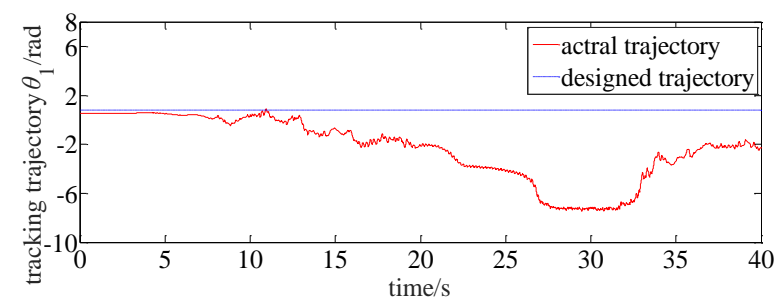

Figure 12: Trajectory tracking of joint 1 angle (closed deadzone).

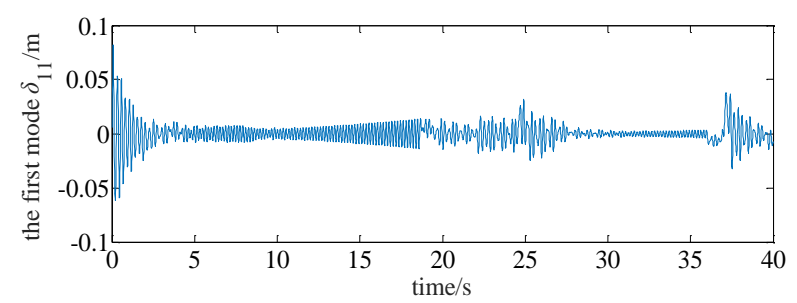

Figure 14: First-order mode of flexible link $B_{1}$ (closed $\boldsymbol{\tau}_{f}$ ).

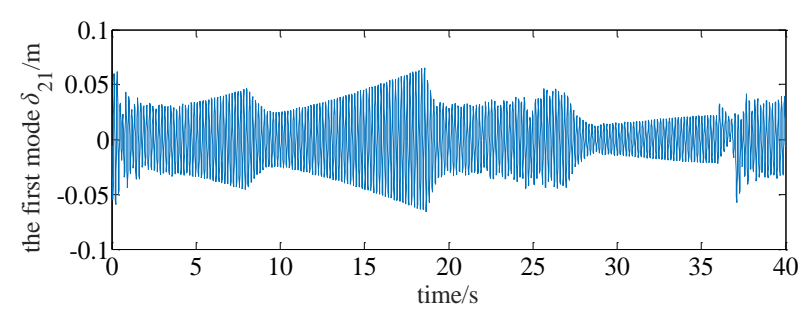

Figure 16: First-order mode of flexible link $B_{2}$ (closed $\boldsymbol{\tau}_{f}$ ).

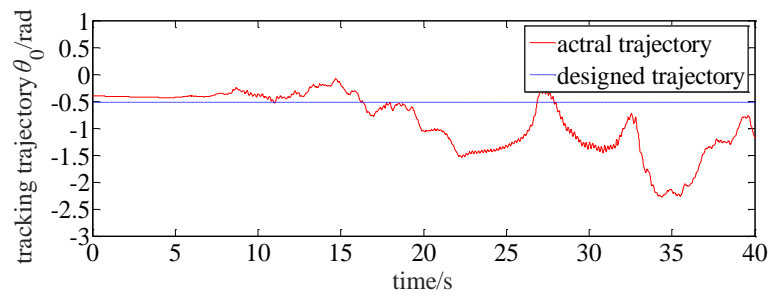

Figure 11: Trajectory tracking of base attitude (closed deadzone).

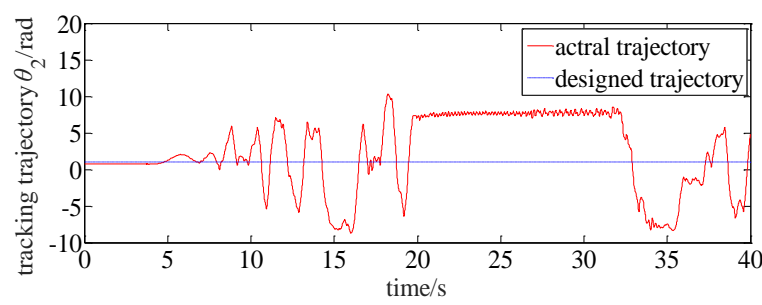

Figure 13: Trajectory tracking of joint 2 angle (closed deadzone).

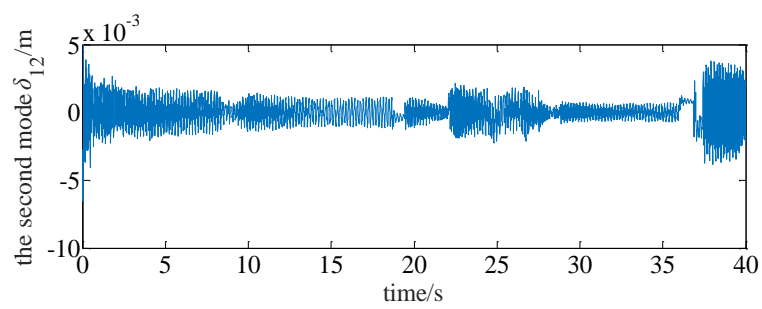

Figure 15: Second-order mode of flexible link $B_{1}$ (closed $\boldsymbol{\tau}_{f}$ ).

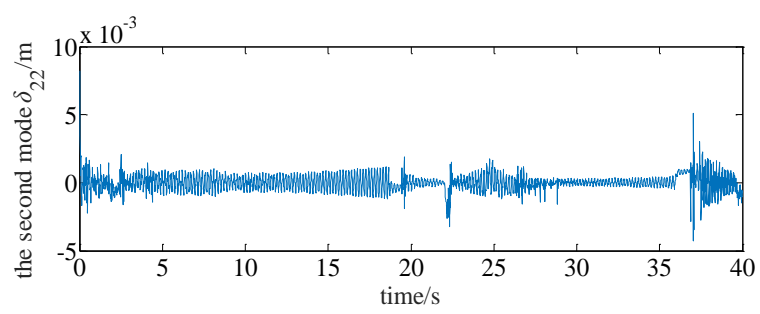

Figure 17: Second-order mode of flexible link $B_{2}$ (closed $\boldsymbol{\tau}_{f}$ ).

The results illustrate that the proposed control method enables the system to perform a rigid desired motion in the presence of deadzone. After the FFSR is adjusted for approximately $20 \mathrm{~s}$, the tracking error of the base attitude is $0.0055 \mathrm{rad}$, and the errors of joints 1 and 2 are 0.0031 and $0.0001 \mathrm{rad}$, respectively. The values of link $B_{1}$ and $B_{2}$ first- and second-order vibration modes are 0.0002 and $0.0012 \mathrm{~m}$ and 0.00007 and $0.00003 \mathrm{~m}$, respectively. These modes basically eliminate vibrations.

Figs. 11 to 13 show the comparison between the desired angular displacements and the actual ones of the base and two joints when the deadzone compensator is closed. The simulations show that the tracking errors are always large and cannot converge at all because the deadzone is uncompensated. The tracking error of the base attitude is up to $3 \mathrm{rad}$, and the errors of joints 1 and 3 are 8 and 10 rad, respectively. As shown in Figs. 4 to 6, turning on the deadzone compensator can effectively decrease the trajectory tracking errors than the turning off it. The actual trajectories of the base and two joints can rapidly and smoothly converge to the desired ones. The validity of the deadzone compensator is thus verified. 
Figs. 14 to 17 show the flexible modes of the two links when $\boldsymbol{\tau}_{f}$ is closed. As shown in these figures, the vibrations cannot be eliminated because they are actively unsuppressed. Vibrations always exist, thereby greatly decreasing the accuracy of trajectory tracking. The maximum values of link $B_{1}$ and $B_{2}$ first- and second-order vibration modes are 0.0375 and $0.0652 \mathrm{~m}$ and 0.0036 and $0.003 \mathrm{~m}$, respectively. Compared with Figs. 7 to 10, the LQR can actively suppress the flexible vibrations with the first- and second-order modes of vibrations converging, thereby improving the accuracy of trajectory tracking and verifying the effectiveness of the vibration suppression control law.

On the basis of the abovementioned simulation results, the deadzone compensator proposed in this study can effectively compensate the adverse effects of asymmetric deadzone on trajectory tracking and the adjustment of its parameter adaptive law is simple. Thus, it has excellent robustness to deadzone. The robust control law can overcome the problem of uncertainties in the dynamic model, thereby realizing the high-precision trajectory tracking of the rigid motion for the slow subsystem. This law does not require the precise dynamic model of the FFSR, or the dynamic equation is linear with respect to inertia parameters. The LQR of the fast subsystem can actively eliminate the vibrations of flexible links and make the flexible-link FFSR complete accurate trajectory tracking.

\section{CONCLUSION}

In order to suppress the vibrations of the flexible links, prevent the influence of deadzone in joint input torque, and realize high-precision trajectory tracking, the dynamic equation of the multi-flexible-link FFSR system was derived, the FFSR was decomposed into fast and slow subsystems on the basis of the singular perturbation theory, and separate control laws were designed. Finally, the simulation experiments of the FFSR with two flexible links were conducted in a MATLAB environment. The following conclusions were drawn:

(1) The robust control method based on nominal model and deadzone estimation compensator of the slow subsystem does not require the dynamic equation to be linear with respect to inertia parameters. This approach can overcome the influence of uncertainty of the dynamic model and deadzone, effectively compensate the input torque deadzone, and guarantee the convergence of tracking errors.

(2) The LQR optimal method of the fast subsystem can actively suppress the flexible vibration of the links.

(3) The proposed scheme can achieve the trajectory tracking and vibration suppression of the FFSR with two flexible links, thereby improving trajectory tracking accuracy.

This study provides an effective scheme for high-precision tracking control of multiflexible-link FFSR with deadzone, which is of great theoretical importance in guiding research on control space robot. However, the deadzone model in this study was simplified, and other parameters, such as deadzone slopes, were not considered. Future research should be conducted to design trajectory tracking control schemes for FFSR with different types of deadzone models.

\section{ACKNOWLEDGEMENT}

This work was supported by the National Nature Science Foundation of China (11372073 and 11072061) and the Fujian Provincial Natural Science Foundation of China (2017J01559). 


\section{REFERENCES}

[1] Flores-Abad, A.; Ma, O.; Pham, K.; Ulrich, S. (2014). A review of space robotics technologies for on-orbit servicing, Progress in Aerospace Sciences, Vol. 68, 1-26, doi: 10.1016/ j.paerosci.2014.03.002

[2] Yoshida, K. (2003). Engineering test satellite VII flight experiments for space robot dynamics and control: theories on laboratory test beds ten years ago, now in orbit, International Journal of Robotics Research, Vol. 22, No. 5, 321-335, doi:10.1177/0278364903022005003

[3] Sabatini, M.; Gasbarri, P.; Monti, R.; Palmerini, G. B. (2012). Vibration control of a flexible space manipulator during on orbit operations, Acta Astronautica. Vol. 73, 109-121, doi: $10.1016 / j$.actaastro.2011.11.012

[4] Kumar, A.; Pathak, P. M.; Sukavanam, N. (2011). Reduced model based control of two link flexible space robot, Intelligent Control and Automation, Vol. 2, No. 2, 112-120, doi:10.4236/ ica.2011.22013

[5] He, W.; David, A. O.; Yin, Z.; Sun, C. (2015). Neural network control of a robotic manipulator with input deadzone and output constraint, IEEE Transactions on Systems, Man \& Cybernetics: Systems, Vol. 46, No. 6, 759-770, doi:10.1109/TSMC.2015.2466194

[6] Sato, K.; Mukai, H.; Tsuruta, K. (2008). An adaptive $H \infty$ control for robotic manipulator with compensation of input torque uncertainty, IFAC Proceedings Volumes (17 $7^{\text {th }}$ IFAC World Congress), Vol. 41, No. 2, 8919-8924, doi:10.3182/20080706-5-KR-1001.01507

[7] Sudharsan, J.; Karunamoorthy, L. (2016). Path planning and co-simulation control of 8 DOF anthropomorphic robotic arm, International Journal of Simulation Modelling, Vol. 15, No. 2, 302-312, doi:10.2507/IJSIMM15(2)9.339

[8] Xie, Y. E.; Wu, X. D.; Shi, Z.; Wang, Z. P.; Sun, J.; Hao, T. W. (2017). The path planning of space manipulator based on Gauss-Newton iteration method, Advances in Mechanical Engineering, Vol. 9, No. 8, 12 pages, doi: $10.1177 / 1687814017718475$

[9] Meng, D. S.; Wang, X. Q.; Xu, W. F.; Liang, B. (2017). Space robots with flexible appendages: dynamic modeling, coupling measurement, and vibration suppression, Journal of Sound and Vibration, Vol. 396, 30-50, doi:10.1016/j.jsv.2017.02.039

[10] Hong, Z.-D.; Yun, C.; Chen, L. (2007). Modeling and trajectory tracking control of a free-floating space robot with flexible manipulators, Robot, Vol. 29, No. 1, 92-96, doi:10.3321/j.issn:1002$\underline{0446.2007 .01 .018}$

[11] Yu, X. Y.; Chen, L. (2015). Singular perturbation adaptive control and vibration suppression of free-flying flexible space manipulators, Proceedings of the Institution of Mechanical Engineers, Part C: Journal of Mechanical Engineering Science, Vol. 229, No. 11, 1989-1997, doi: $10.1177 / 0954406214551777$

[12] Zhang, L.; Liu, J. (2012). Observer-based partial differential equation boundary control for a flexible two-link manipulator in task space, IET Control Theory \& Applications, Vol. 6, No. 13, 2120-2133, doi:10.1049/iet-cta.2011.0545

[13] Yang, X.; Ge, S. S.; He, W. (2018). Dynamic modelling and adaptive robust tracking control of a space robot with two-link flexible manipulators under unknown disturbances, International Journal of Control, Vol. 91, No. 4, 969-988, doi:10.1080/00207179.2017.1300837

[14] Cheong, J.; Chung, W. K.; Youm, Y. (2001). Fast suppression of vibration for multi-link flexible robots using parameter adaptive control, Proceedings of 2001 IEEE/RSJ International Conference on Intelligent Robots and Systems, Vol. 2, 913-918, doi:10.1109/IROS.2001.976285

[15] Masoudi, R.; Mahzoon, M. (2011). Maneuvering and vibrations control of a free-floating space robot with flexible arms, Journal of Dynamic Systems, Measurement, and Control, Vol. 133, No. 5, Paper 051001, 8 pages, doi:10.1115/1.4004042

[16] Han, S.-I.; Lee, K.-S.; Park, M.-G.; Lee, J.-M. (2011). Robust adaptive deadzone and friction compensation of robot manipulator using RWCMAC network, Journal of Mechanical Science and Technology, Vol. 25, No. 6, Paper 1583, doi:10.1007/s12206-011-0328-9

[17] Cheong, J. Y.; Han, S. I.; Lee, J. M. (2013). Adaptive fuzzy dynamic surface sliding mode position control for a robot manipulator with friction and deadzone, Mathematical Problems in Engineering, Vol. 2013, Paper 161325, 15 pages, doi: $\underline{10.1155 / 2013 / 161325}$ 
[18] Hsu, C.-F.; Kuo, T.-C. (2014). Intelligent complementary sliding-mode control with dead-zone parameter modification, Applied Soft Computing, Vol. 23, 355-365, doi: $\underline{10.1016 /}$ j.asoc. 2014.06 .008

[19] Han, S. I.; Lee, J. M. (2016). Finite-time sliding surface constrained control for a robot manipulator with an unknown deadzone and disturbance, ISA Transactions, Vol. 65, 307-318, doi: $10.1016 /$ j.isatra.2016.07.013

[20] Liu, Y. Z.; Chen, L. Q.; Chen, W. L. (2011). Vibration Mechanics, $5^{\text {th }}$ ed., Higher Education Press, Beijing

[21] Wang, Z. H.; Yang, B.; Cheng, L.; Zhang, S. S. (2006). Robust adaptive deadzone compensation of DC servo system, IEEE Proceedings - Control Theory and Applications, Vol. 153, No. 6, 709713, doi:10.1049/ip-cta:20050253 